\title{
Near Tip Stress and Strain Fields for Short Elastic Cracks
}

\author{
by \\ A.H. Soediono*, G.A. Kardomateas** and R.L. Carlson***

\begin{abstract}
Recent experimental fatigue crack growth studies have concluded an apparent anomalous behavior of short cracks. To investigate the reasons for this unexpected behavior, the present paper focuses on identifying the crack length circumstances under which the requirements for a single parameter ( $K_{I}$ or $\Delta K_{I}$ if cyclic loading is considered) characterization are violated. Furthermore, an additional quantity, the $T$ stress, as introduced by Rice, and the related biaxiality ratio, $B$, are calculated for several crack lengths and two configurations, the single-edge-cracked and the centrally-cracked specimen. It is postulated that a two-parameter characterization by $K$ and $T$ (or $B$ ) is needed for the adequate description of the stress and strain field around a short crack. To further verify the validity of this postulate, the influence of the third term of the Williams series on the stress, strain and displacement fields around the crack tip and in particular on the $B$ parameter is also examined. It is found that the biaxiality ratio would be more negative if the third term effects are included in both geometries. The study is conducted using the finite element method with linearly elastic material and isoparametric elements and axial (mode I) loading. Moreover, it is clearly shown that it is not proper to postulate the crack size limits for "short crack" behavior as a normalized ratio with the specimen width, $a / w$; it should instead be stated as an absolute, or normalized with respect to a small characteristic dimension such as the grain size. Finally, implications regarding the prediction of cyclic (fatigue) growth of short cracks are discussed.
\end{abstract}

\section{Introduction}

The short fatigue crack problem consists essentially of defining an alternative formulation that accounts for the observation that small cracks can propagate at rates different from the corresponding ones for long cracks at the same nominal stress intensity factors. In general, short cracks under cyclic load are observed to grow at stress intensities below threshold; some extend with decaying growth rate until arrest, while others propagate

\footnotetext{
* Research Engineer, Agency for The Assessment and Application of Technology, Indonesia.

** Associate Professor, School of Aerospace Engineering, Georgia Tech:

*** Professor Emeritus, School of Aerospace Engineering, Georgia Tech.
} 
quite rapidly to merge with long crack behavior as shown in Figure 1. A resolution of the problem therefore has practical significance. First, it could enhance the present damage tolerance procedures. Furthermore, as overall life is mostly taken up by short crack growth, predicting the accelerated and sub-threshold extension of small flaws can lead to alternative, more accurate methods for assessing fatigue life.

Linear Elastic Fracture Mechanics (LEFM) theory is applied when the radius of the plastic zone is small compared to the critical dimensions of the body, as codified in both British and American Standards. If $\sigma_{Y}$ is the yield stress, $a$ is the crack length, $w$ is the width, $w-a$ is the ligament and $t$ is the thickness, these standards require:

$$
w, w-a, \quad t \geq 2.5\left(\frac{K}{\sigma_{Y}}\right)^{2}
$$

where $K$ is the stress intensity factor.

To describe the basis for the above premise, let $r, \theta$ be polar coordinates centered at the tip of a crack in a body under plane strain deformation. The small strain linear elastic solution results in stresses of the form

$$
\sigma_{i j}=K r^{-1 / 2} f_{i j}(\theta)+\text { non }- \text { singular terms }
$$

near the crack tip, where the set of functions $f_{i j}$ is normalized so that the singular part of the stress acting ahead of the tip, normal to the plane of the crack, is $K(2 \pi r)^{-1 / 2}$. The small scale yielding approximation then incorporates the notion that, even though Equation (2) is inaccurate within and near a small crack tip yield zone, its dominant singular term should still govern the deformation state within that zone. Hence, the actual elastic-plastic problem is replaced by a problem formulated in boundary layer style. As is often said, the small yield zone is "surrounded" by the dominant elastic singularity, and the applied loadings and geometric dimensions and shape of the body influence conditions within the plastic region only insofar as they enter the formula for $K$, as computed elastically.

A consequence of this formulation is that the plastic zone dimension, $r_{p}$ and the crack 
tip opening displacement $\delta_{t}$, are given by formulae of the type

$$
r_{p}=\alpha K^{2} / \sigma_{0}^{2}, \quad \delta_{t}=\beta K^{2} / E \sigma_{0}
$$

where $E$ is the elastic tensile modulus, $\sigma_{0}$ is the yield strength, and $\alpha, \beta$ are dimensionless factors which may, for example, depend on Poisson's ratio, strain-hardening exponent, etc., but are independent of the applied load and specimen geometry.

The plastic zone size from Equation (3) establishes a geometric dimension indicating the region over which deviations from elastic behavior occur. Rice [1] was the first to recognize that, since the crack length is a characteristic geometric dimension associated with the elastic stress field, a correction to Equation (3) is required when the length predicted by Equation (3) is comparable to or greater than the crack length, i.e. the stress intensity factor may no longer be expected to control the plasticity.

When the size of the plastic zone becomes large compared to the crack length, which is one of the characteristic dimensions, the requirements of LEFM may be invalidated. A similar question obviously exists for J-dominance. In other words, there are conditions under which a single parameter characterization of the crack tip field cannot be claimed. Indeed, it is known that for full plasticity the asymptotic plastic field is not unique but is instead a function of the geometry, loading mode and strain hardening rate and singleparameter characterization models cannot account for the differences in propagation rates from fully plastic crack growth tests (Kardomateas and McClintock, [2]).

To further examine this notion, consider keeping the 'non-singular' terms of Equation (2). Recently, Carlson and Saxena [3] have calculated the stresses due to the second term. In another work, using the analyses of Williams [4], Rice [5] defined the $T$-stress from the second term of the expansion of Equation (2) as follows:

$$
\left[\begin{array}{ll}
\sigma_{x x} & \tau_{x y} \\
\tau_{y x} & \sigma_{y y}
\end{array}\right]=\frac{K}{\sqrt{r}}\left[\begin{array}{ll}
f_{x x}(\theta) & f_{x y}(\theta) \\
f_{y x}(\theta) & f_{y y}(\theta)
\end{array}\right]+\left[\begin{array}{ll}
T & 0 \\
0 & 0
\end{array}\right]+\begin{aligned}
& \text { terms which vanish } \\
& \text { at crack tip }
\end{aligned}
$$

Here $(x, y)$ is the plane of straining and the crack coincides with the $x$ - axis, so it is seen 
that the portion of the non-singular stress field which does not vanish at the tip amounts to a uniform stress $\sigma_{x x}=T$ acting parallel to the crack plane.

The above representation raises the issue of a two-parameter boundary layer formulation for short cracks, in which Equation (2) is replaced by the requirement of an asymptotic approach to the field given by the two leading terms of Equation (4).

In the same context, Leevers and Radon [6] introduced a biaxiality parameter $B$ that relates the magnitude of the $T$-stress:

$$
B=\frac{T \sqrt{\pi a}}{K}
$$

In terms of $B$, the displacement field can be written in the form:

$$
\begin{aligned}
& u_{x x}=\frac{K}{E}(1+\nu) \sqrt{\frac{r}{2 \pi}} f_{x x}(\theta)+\left(1-\nu^{2}\right) \frac{B}{E \sqrt{\pi a}} K r \cos \theta \\
& u_{y y}=\frac{K}{E}(1+\nu) \sqrt{\frac{r}{2 \pi}} f_{y y}(\theta)-\nu(1+\nu) \frac{B}{E \sqrt{\pi a}} K r \sin \theta .
\end{aligned}
$$

The biaxiality parameter $B$ must be calculated for each particular specimen geometries to correlate the full field solutions with the two term representations. The displacements given above or the stress field expressions (4) can serve in that purpose. Concerning the displacements, it should be noted that on the crack flanks $\phi=\pi$, the angular functions $f_{i}(\phi)$ are zero, allowing the biaxiality parameter to be calculated by direct inspection of the asymptotic displacements given by Equation (6) and stress fields given by Equation (4). Such methods have already been used by Betegon and Hancock [7].

The above discussion summarizes the work-to-date on extending the applicability of LEFM to include cases that necessitate the inclusion of correction terms such as the present case of short cracks.

Elastic-plastic fracture mechanics, on the other hand, is based on the HRR singularity, named after Hutchinson[8], Rice and Rosengren [9], which describes the asymptotic stress and strain fields in terms of the strain hardening exponent $n$ within the plastic zone under 
small-scale yielding conditions as follows:

$$
\begin{aligned}
\sigma_{i j} & =Y\left[\frac{J}{\epsilon_{0} Y \alpha I_{n} r}\right]^{1 /(n+1)} \tilde{\sigma}_{i j}(\phi, n), \\
\epsilon_{i j} & =\frac{Y a}{E}\left[\frac{J}{\epsilon_{0} Y \alpha I_{n} r}\right]^{n /(n+1)} \tilde{\epsilon}_{i j}(\phi, n),
\end{aligned}
$$

where $\tilde{\sigma}_{i j}(\phi, n)$ and $\tilde{\epsilon}_{i j}(\phi, n)$ and $I_{n}$ are tabulated functions. In this context the stresses and strains are uniquely characterized by the $J$ quantity. Elastic-plastic asymptotic crack tip fields, being single parameter characterizations, would naturally have to be investigated regarding their extent of validity in the same context as the LEFM, i.e. the question of $J$-dominance or, in other words, that of a single parameter characterization of crack tip fields for short cracks.

Achieving crack growth prediction capabilities for short cracks can lead to formulations for predicting fatigue crack growth. Indeed, fatigue, crack nucleation and growth pass through distinct regimes which can be characterized by crack length. The relation of the fatigue failure loading boundary to crack length can be very nicely illustrated by use of the Kitagawa diagram [10] shown in Figure 1. In the plot of stress range versus crack length, the boundary is divided into three regimes which are depicted as two straight lines in regimes I and III and a curve in regime II. Stress range values below the boundary correspond to cases in which cracks are arrested. Above the boundary, crack growth occurs.

The ordinate value of the boundary in regime I corresponds to the endurance limit. The line in regime III represents the value of stress range, $\Delta \sigma$, corresponding to the threshold value of the stress intensity range, $\Delta \sigma$, in the relation

$$
\Delta K=Y \Delta \sigma \sqrt{\pi a}
$$

$\mathrm{Y}$ is constant for the given crack configuation and $a$ is the crack length.

If the dashed lines were extended and used as the boundary in regime II, predictions would be nonconservative because cracks are observed to grow below these lines. The 
boundary in regime II is, therefore, represented by the solid curve connecting the two straight lines. Short crack behavior is characterized in Figure 1 in regime II; hence the primary emphasis here will be on regime II.

The main objective of this work is to (a) determine the crack lengths at which the $K$ singularity cannot predict the asymptotic behavior, (b) determine the $T$ or $B$ parameter for these short cracks and (c) examine the influence of the third term of the asymptotic expansion of the stress field on the calculated $B$ values. The Center-Cracked $(\mathrm{CC})$ and Single Edge-Cracked (SEC) specimens are used for this purpose. They are subjected to monotonic tension stress. The finite element program, MSC/Nastran, is used for this computation.

\section{Formulation of Non-Singular Parameters}

Prediction methodologies in fracture mechanics are mainly based on the proper characterization of the crack-tip stress and strain field. Expanding the stress field in cylindrical coordinates $(r, \theta)$ about the crack tip, following the work of Williams [4],

$$
\sigma_{i j}=\frac{A_{1}}{\sqrt{r}} f_{i j}^{1}(\theta)+A_{2} f_{i j}^{2}+A_{3} \sqrt{r} f_{i j}^{3}+\ldots
$$

gives the first term singular at the crack tip and the remaining terms being finite and bounded. In classical linear elastic fracture mechanics, the characterization is centered around the stress intensity factor $K$, i.e. the first term. Elastic fracture mechanics is thus based on the premise that fracture processes which occur close to the crack tip are only determined by the first term in the expansion, allowing the asymptotic elastic stress field of a symmetrically loaded mode I crack to be expressed in the form

$$
\sigma_{i j}=\frac{K_{I}}{\sqrt{2 \pi r}} f_{i j}(\theta)
$$

Proposals have been made to provide a correction to plastic zone size estimates by extending elastic solutions to include non-singular terms of the Williams series [4]. In Equations (4), Rice [5] defined the $T$ stress for the second term of the series; this equation 
raises the issue of a two parameter boundary layer formulation for short cracks in which stresses are determined by an asymptotic approach to the field given by the two leading terms of $K$ and $T$.

In the same context, Leevers and Radon [6] introduced a biaxiality parameter $B$ that relates the magnitude of the $T$ stress as in Equation (5). In terms of $B$ the stress and displacement field can be written in the form:

$$
\begin{gathered}
\sigma_{r r}=\frac{K_{I}}{\sqrt{2 \pi r}}\left(\frac{5}{4} \cos \frac{\theta}{2}-\frac{1}{4} \cos \frac{3 \theta}{2}\right)+\frac{K_{I}}{\sqrt{\pi a}} B \cos ^{2} \theta \\
\sigma_{\theta \theta}=\frac{K_{I}}{\sqrt{2 \pi r}}\left(\frac{3}{4} \cos \frac{\theta}{2}+\frac{1}{4} \cos \frac{3 \theta}{2}\right)+\frac{K_{I}}{\sqrt{\pi a}} B \sin ^{2} \theta \\
\sigma_{r \theta}=\frac{K_{I}}{\sqrt{2 \pi r}}\left(\frac{1}{4} \sin \frac{\theta}{2}+\frac{1}{4} \sin \frac{3 \theta}{2}\right)-\frac{K_{I}}{\sqrt{\pi a}} B \sin \theta \cos \theta \\
u_{r r}=\frac{K_{I} \sqrt{2 \pi r}}{8 \pi \mu}\left[(2 \kappa-1) \cos \frac{\theta}{2}-\cos \frac{3 \theta}{2}\right]-\frac{K_{I}(r-a)}{2 \mu \sqrt{\pi a}} B\left(\cos ^{2} \theta-\nu\right) \\
u_{\theta \theta}=-\frac{K_{I} \sqrt{2 \pi r}}{8 \pi \mu}\left[-(2 \kappa+1) \sin \frac{\theta}{2}+\sin \frac{3 \theta}{2}\right]-\frac{K_{I} r}{2 \mu \sqrt{\pi a}} B\left(\frac{1}{2} \sin 2 \theta\right)
\end{gathered}
$$

where $\mu$ is the shear modulus and $\kappa=3-4 \nu$ for plane strain and $\kappa=(3-\nu) /(1+\nu)$ for plane stress. Furthermore, a model for plane strain yielding by Rice [5] results in the following expressions for plastic zone size and crack opening displacement:

$$
\begin{aligned}
& r_{p}=\frac{\pi}{18}\left(\frac{K_{I}}{\sqrt{3} \tau_{Y}}\right)^{2}\left[1-\frac{4}{3} \sqrt{\frac{2}{3}}\left(\frac{T}{\sqrt{3} \tau_{Y}}\right)+. . \cdot\right. \\
& \delta_{t}=\frac{16}{27} \sqrt{\frac{2}{3}} \frac{\left(1-\nu^{2}\right) K_{I}^{2}}{E\left(\sqrt{3} \tau_{Y}\right)}\left[1-\frac{2}{3} \sqrt{\frac{2}{3}}\left(\frac{T}{\sqrt{3} \tau_{Y}}\right)+\ldots\right],
\end{aligned}
$$

where $\tau_{Y}$ is the shear yield stress. The second terms inside the brackets represent the deviation from the small scale yielding approximation. Note that $K$ and $T$ are directly proportional to the applied loading.

The stress intensity factor, $K$, which was introduced in Equation (2), defines the magnitude of the local stresses near the crack tip. This factor depends on loading, crack 
size, crack shape, and geometric boundaries. Closed-form solutions for $K$ have been derived for a number of simple configurations. For more complex situations, the stress intensity factor can be estimated by experiment or numerical analysis. The Stress Intensity Factor solutions for a single edge-cracked (SEC) and a center-cracked (CC) configuration under mode I loading, which are the object of our investigation, are [11]:

$$
K_{I_{S E C}}=\left[1.12-0.23 a / w+10.6(a / w)^{2}-21.7(a / w)^{3}+30.4(a / w)^{4}\right] \sigma_{\infty} \sqrt{\pi a}
$$

and

$$
K_{I_{C C}}=\left\lceil\frac{1-0.5 a / w+0.326(a / w)^{2}}{\sqrt{1-a / w}}\right\rceil \sigma_{\infty} \sqrt{\pi a}
$$

where $w$ is the specimen width.

Numerical Analysis and Finite Element Models. Single-edge-cracked (SEC) and centercracked $(\mathrm{CC})$ bars with $(a / w)$ ratios of 0.02 through 0.1 , where $a$ is the crack length or half crack length, respectively, and $w$ the width of the specimen, were considered. Concerning the other geometric dimensions, the specimens had a thickness, $t$ and height, $h$, characterized by the dimensionless parameters $t / w=0.08$, and $h / w=2.0$ for the CC specimen and ,4.0 for the SEC specimen, respectively.

The models were meshed with eight-noded quadrilateral and six-noded triangular plane strain elements provided by the finite element code MSC-Nastran [12]. These 2-D finite element grids are indicated in Figure 2. The models were force loaded on the remote boundary by a uniform tensile stress in the y-direction. The crack tip was modeled by a focused mesh with initially coincident but independent crack tip nodes. Angular spacings of 15 degrees and minimum radial spacings of $0.05 \mathrm{~mm}$ for every sector were used. Linear elastic material with Young's modulus $E=72 \mathrm{GPa}$ and Poisson's ratio, $\nu=0.3$ was assumed; the yield stress, $\sigma_{Y}$, is taken to be such that $E / \sigma_{Y}=176.0$. The mesh configuration for each specimen consists of a total of 235 elements. Furthermore, 
force balance studies were also conducted to check the applied and reaction forces on each specimen in order to ensure that the mesh is performing in a satisfactory manner.

\section{Results and Discussion}

In order to correlate with the results of Leever and Radon [6] we shall give both the ratio $a / \rho$ and $a / w$ in presenting the results. Denote by $\rho$ the typical grain size, $\rho=0.05$ $\mathrm{mm}$. The stress fields ahead of the crack are shown in Figures 3 and 4 for $a / \rho$ ratios of $10,20,50$ and $200(a / w=0.02,0.04,0.1,0.4$, respectively) where they are compared with the theoretical LEFM field, calculated from Equation (10). The stresses are nondimensionalized with the remote stress $\sigma_{\infty}$, while the original distance, $r$ of a point ahead of the crack is non-dimensionalized with the grain size $\rho$. It is seen that for these crack lengths the stresses are above the LEFM values, the discrepancy increasing with the smaller crack lengths. Beyond $a / \rho=50(a / w=0.1)$, the stresses begin to converge to the LEFM predictions. Notice that Leever and Radon [6] results were for $a / w$ values above 0.2 ; therefore, the present work, among others, complements the latter one.

In order to elucidate the size issue, three center-cracked specimens which have the same $a / w$ ratio of 0.02 but different absolute sizes have been examined; they consist of a short $\operatorname{crack}(a / \rho=6)$ and long $\operatorname{cracks}(a / \rho=60$ and 300) in respectively smaller and larger specimens that preserve the ratios of crack length to other dimensions. From Figure 5 it is clearly seen that the stresses follow the LEFM prediction for the long cracks, but there is a distinct discrepancy for the short crack, i.e. the LEFM prediction is no longer valid. This example shows that $a / w$ is not the proper quantity to express the size requirements for "short crack" behavior.

Let us require that the stress ahead the crack tip must be within $10 \%$ of the LEFM field at a chosen distance from the tip for LEFM dominance criteria. It can be seen that at a distance of $r / \rho=2$ ahead the crack tip, Figures 3a,b show that the crack tip stress field $\sigma_{\theta \theta}$ for the center-cracked specimens differs significant from the results of Equation (10) for short crack lengths; but for a longer crack, the stresses from FEM and Equation 
(10) have a tendency to match, as shown in these same Figures. A similar situation also occurs in the SEC specimens, but the distance ahead the crack tip is shorter $(r / \rho=1.5)$; they are presented in Figure 4.

The biaxiality parameter $B$ (or alternatively, the $T$-stress) was calculated for each of the geometries studied. The simplest and most direct method of calculating the $T$-stress involves inspection of the stress or displacement fields associated with the crack. Leevers and Radon [6] have introduced a more refined method for calculating $B$ for the centercracked specimens; they essentially adjusted the centerline traction individually according to the deviation of the calculated displacement. In this work the stress field of Equation (4) was used to calculate $B$ from $\sigma_{x x}$.

Figures $6 \mathrm{a}, \mathrm{b}$ show that the center-cracked specimens consistently have higher negative $B$ values than the single-edge-cracked ones. The implied compressive $T$ stresses are induced by the constraint against in-plane bending on the centerline; they are responsible for the excellent directional stability of internal cracks [6]. By using a linear aprroximation, for values of crack length less than $0.1 w$ or $50 \rho, B$ can be expressed by:

$$
B=-[1+0.082(a / w)]
$$

or

$$
B=-\left[1+1.6510^{-4}(a / \rho)\right] .
$$

The finite-width effect does not drastically modify $B$ values, which remain about -1 as $2 a / w$ goes to zero. Equation (15a) is slightly different than the one in Reference [6] (where there was a factor of 0.085 instead of the 0.082 here). In Ref. 6 a linear approximation was used to determine $B$ values for crack-lengths $a / w$ greater than 0.2 , but in this research we have considered crack-lengths less than $0.2(a / \rho<100)$.

$B$ values for the single-edge-cracked specimens are quite different than those of the center-cracked specimens. By using a curve fitting of polynomial third order, the $B$ values 
for the SEC specimens can be expressed by:

$$
B=-0.52-1.50(a / w)+12.70(a / w)^{2}-20.70(a / w)^{3}
$$

or in term of $a / \rho$, it also can be written as

$$
B=-0.52-4.1710^{-3}(a / \rho)+8.9110^{-5}(a / \rho)^{2}-6.2510^{-7}(a / \rho)^{3} .
$$

Figure $6 \mathrm{~b}$ shows that the $B$ values for the single-edge-cracked case are almost one-half those of the center-cracked specimen (as the crack length goes to zero).

Figure 7 shows the sensitivity with respect to number of elements of the stress biaxiality ratio, $B$, for both specimens. It can be seen that $B$ has a stable value when the number of elements is above 225. Also Reference [6] has indicated that small values of the height, $h / w$ can have an influence on determining $B$; an example for the center-cracked specimen showed that $B$ has the tendency to be constant for values of $h / w=1.2$ to 2.0 for $2 a / w=$ 0.5 .

Figure 8 shows that the crack tip displacement field in the $\theta$-direction for the singleedge-cracked specimens does not fit with the displacement field given by the $K$-singularity at the distance between 0 and 20 grains size behind the crack tip when the crack is short, but for longer cracks the displacement field gradually fits. Similar situations also occur in the center-cracked specimens, as shown in Figure 9. This confirms again the breakdown of the LEFM singularity for short cracks.

The Effect of the Third Term. An evaluation of including the effect of the third term on the biaxiality ratio, $B$, can be conducted by examining the stress distribution, $\sigma_{\theta \theta}(r, 0)$ on the plane in front of the crack tip. Specifically, the Williams series can be written up to the third term:

$$
\sigma_{\theta \theta}(r, 0)=\frac{K_{I}}{\sqrt{2 \pi r}}+3 C_{3} r^{1 / 2}
$$

i.e. $\sigma_{\theta \theta}$ includes the third term, $C_{3}$, but not the second, (or $B$ ) term. 
On the other hand, up to the third term,

$$
\sigma_{r r}(r, 0)=K_{I}\left(\frac{1}{\sqrt{2 \pi r}}+\frac{B}{\sqrt{\pi a}}\right)+3 C_{3} r^{1 / 2}
$$

i.e. $\sigma_{r r}$ includes the second, (or $\left.B\right)$ term as well as the third $\left(C_{3}\right)$ term.

Therefore, eq (17) can be used to calculate $C_{3}$ (from the finite element data on $\sigma_{\theta \theta}$ ) and then the calculated value of $C_{3}$ can be used in eq (18) to find the biaxiality ratio $B$ with the third term effects now included.

Fig. 10 shows the effect of the third term on the biaxiality ratio for the center-cracked geometry and Fig. 11 for the single-edge-cracked geometry. It seems that the third term requires a larger correction to the biaxiality ratio for short crack length and has a relatively small effect for long crack length. The biaxiality ratio would be more negative (higher in absolute value) if the third term effects are included in both geometries. Fig. 12 shows the percentage increase, $\Delta B=\left(B_{\text {incl }}-B_{\text {not incl }}\right) / B_{\text {not incl }}$, which is seen to increase rapidly as $a / \rho \rightarrow 0$ (for a typical short crack $a / \rho=10$ in the center-cracked case this is moderate, about 15\%). However, it should be noted that what constitutes a significant contribution of a neglected term to the fracture behavior may depend on factors other than the percentage error. For example, although the percentage error due to the third term may appear to be moderate for typical short crack sizes, the fatigue crack growth rate in the near threshold region is drastically affected by very small changes in the value of $\Delta K$.

By using curve fittings from the results of Figs. 10 and 11, the stress biaxiality ratio equations with the third term effects included can be presented as

$$
B=-\left[1+1.6510^{-4}(a / \rho)+0.49(a / \rho)^{-0.5}\right]
$$

for CC specimens and

$$
B=-0.72-1.8510^{-3}(a / \rho)+1.0510^{-6}(a / \rho)^{2}-8.9610^{-9}(a / \rho)^{3} .
$$

for SEC specimens. These can be compared with the equations (15b) and (16b), respectively, where the third term effects are not included. 
Implications for Short Fatigue Crack Growth. As has been already stated, an extended representation of fatigue crack growth behavior may be developed by considering the Kitagawa diagram (Fig. 1) for the case in which the minimum stress is zero. Then the stress range is equal to the maximum stress, and an upper bound boundary corresponding to the fracture toughness can be included as depicted by the upper, dashed curve. The map between the boundaries may, in turn, be divided into two regions corresponding to a linear elastic fracture mechanics [LEFM] response and a nonlinear inelastic fracture mechanics response [NIFM].

Short crack behavior is characterized in Figure 1 in regime II. Serious consideration of the behavior of short fatigue cracks began with results published in 1975 by Pearson [13]. It should, however, be noted that Rice [1] had predicted earlier that the stress intensity factor may no longer be expected to control the local field for crack lengths comparable to the plastic zone size.

The behavior observed has been described as "anomalous", because crack growth for short cracks was observed to occur at values of stress intensity factor range below the threshold value.

The results of this paper indicate that the anomalous designation may be inappropriate, and it may indeed be a consequence of the use of correlation procedures which are not valid. In fact, a clarification of short fatigue crack growth behavior appears to require that two mechanisms must be considered. The first one, which was considered in this paper, involves the fact that the requirement of small scale yielding for the use of stress intensity factor range as a correlation parameter is not satisfied for short cracks (i.e. LEFM is not valid). The other, which needs to be considered in the future, concerns the role of obstruction to closure upon unloading. For a given range of stress intensity factor, obstruction to closure is generally less for short cracks than for long cracks.

Although most attention has been directed toward consideration of these two mechanisms, a third potentially important mechanism should be mentioned. It has been found 
that short fatigue cracks in steels subjected to chemically active environments grow faster than long cracks for the same $\Delta K[14,15]$. Since corrosion fatigue failures are often observed in service, this behavior should not be ignored.

A review of the literature on the growth of short fatigue cracks indicates that the research studies that have been reported have usually been motivated by one of two distinct goals. The primary goal of material scientists has been to use the results of their studies to contribute to an understanding of the fundamental mechanisms of short crack growth. Mechanics specialists have been motivated primarily by the need to develop design codes. There is a need for more collaboration between researchers from these two groups. The paper by Blom, et al [16] examines both mechanics and metallurgical issues and it provides valuable insight into the interacting aspects of the short crack growth problem.

\section{Acknowledgement}

The authors acknowledge that the studies which led to the preparation of this paper were initiated during the support of the Warner Robins Air Logistics Center, Robins AFB under Contract No: F09603-85-G-3104-0034. They are also grateful for the interest and encouragement provided by Gary Chamberlain.

\section{References}

1. J.R. Rice, in Fatigue Crack Propagation ASTM STP 415 (1967) 247-309.

2. G.A. Kardomateas and F.A. McClintock, International Journal of Fracture 35 (1987) 103-124.

3. R.L. Carlson and A. Saxena, International Journal of Fracture 33 (1987) 37-39.

4. M.L. Williams, Journal of Applied Mechanics 24 (1957) 109-114.

5. J.R. Rice, Journal of the Mechanics and Physics of Solids 22 (1974) 17-26.

6. P.S. Leevers and J.C. Radon, International Journal of Fracture (1983) 311-324.

7. C. Betegon and J.W. Hancock, Journal of Applied Mechanics 58 (1991) 104-110.

8. J.W. Hutchinson, Journal of the Mechanics and Physics of Solids 16 (1968) 13-31. 
9. J.R. Rice and G.F. Rosengren, Journal of the Mechanics and Physics of Solids 16 (1968) 1-12.

10. H. Kitagawa and S. Takahashi, Proceedings ICM-2 ASM (1976) 627-631

11. Kåre Hellan, Introduction to Fracture Mechanics McGraw-Hill Book Co., New York (1984).

12. MSC/NASTRAN User's Manual, Version 67, McNeal-Schwindler Corporation Vol. 1 and 2 (1991).

13. S. Pearson, Engineering Fracture Mechanics 7 (1975) 235-247.

14. Y. Nakai and J. Ohji, Proceedings Fourth International Conference on Fatigue and Fatigue Thresholds (1990) 1605-1610.

15. M. Nakajima Y. Kato and Y. Kitaoka, ibid 1611-1616.

16. A.F. Blom, A. Hedlund, W. Zhao, A. Fathulla, B. Weiss, and R. Stickler, The Behavior of Short Fatigue Cracks (K.J. Miller and E.R. delosRios editors) Mechanical Eng. Public. (1986) 37-66. 


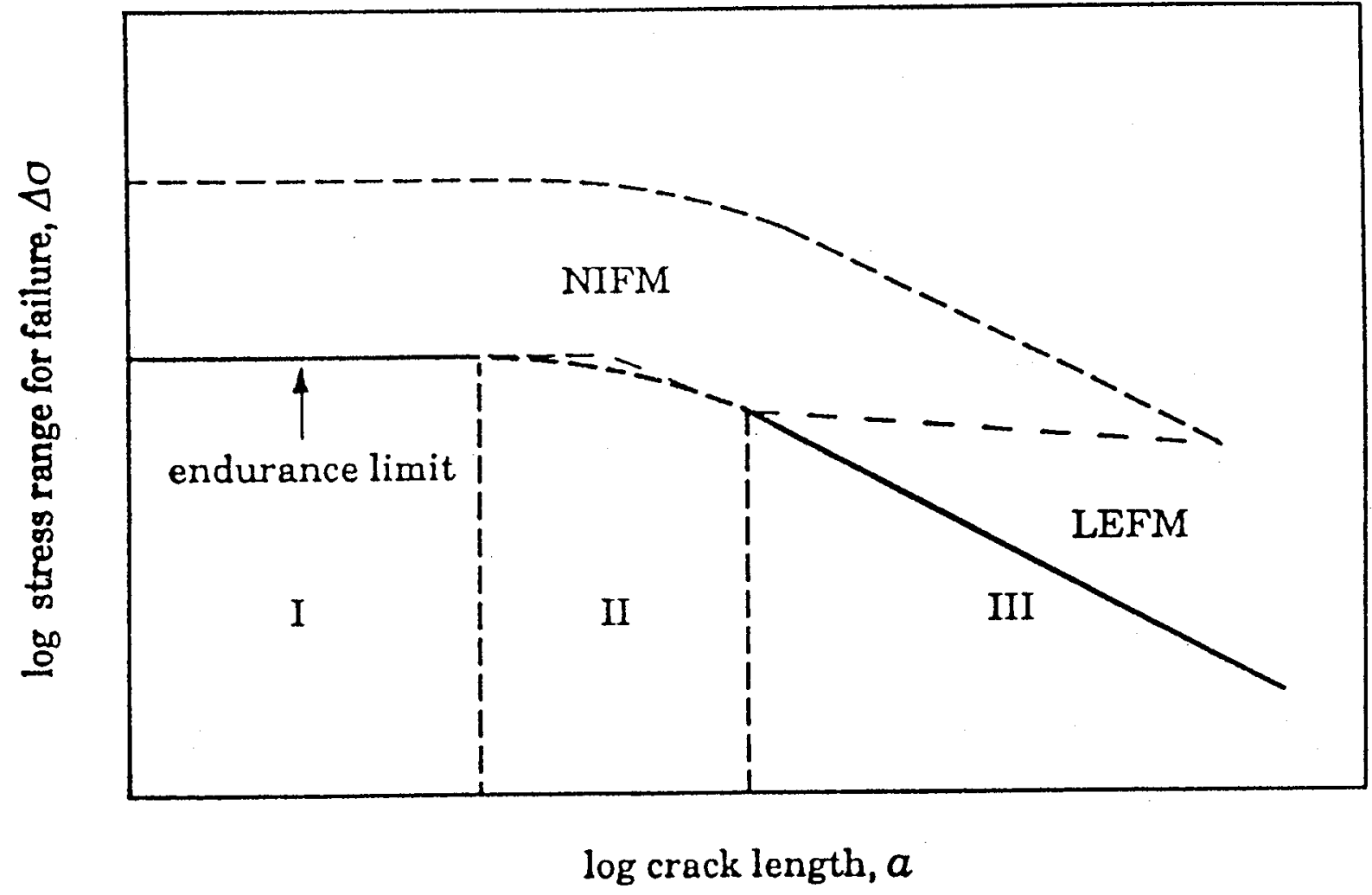

Fig. 1. The Kitagawa diagram. 

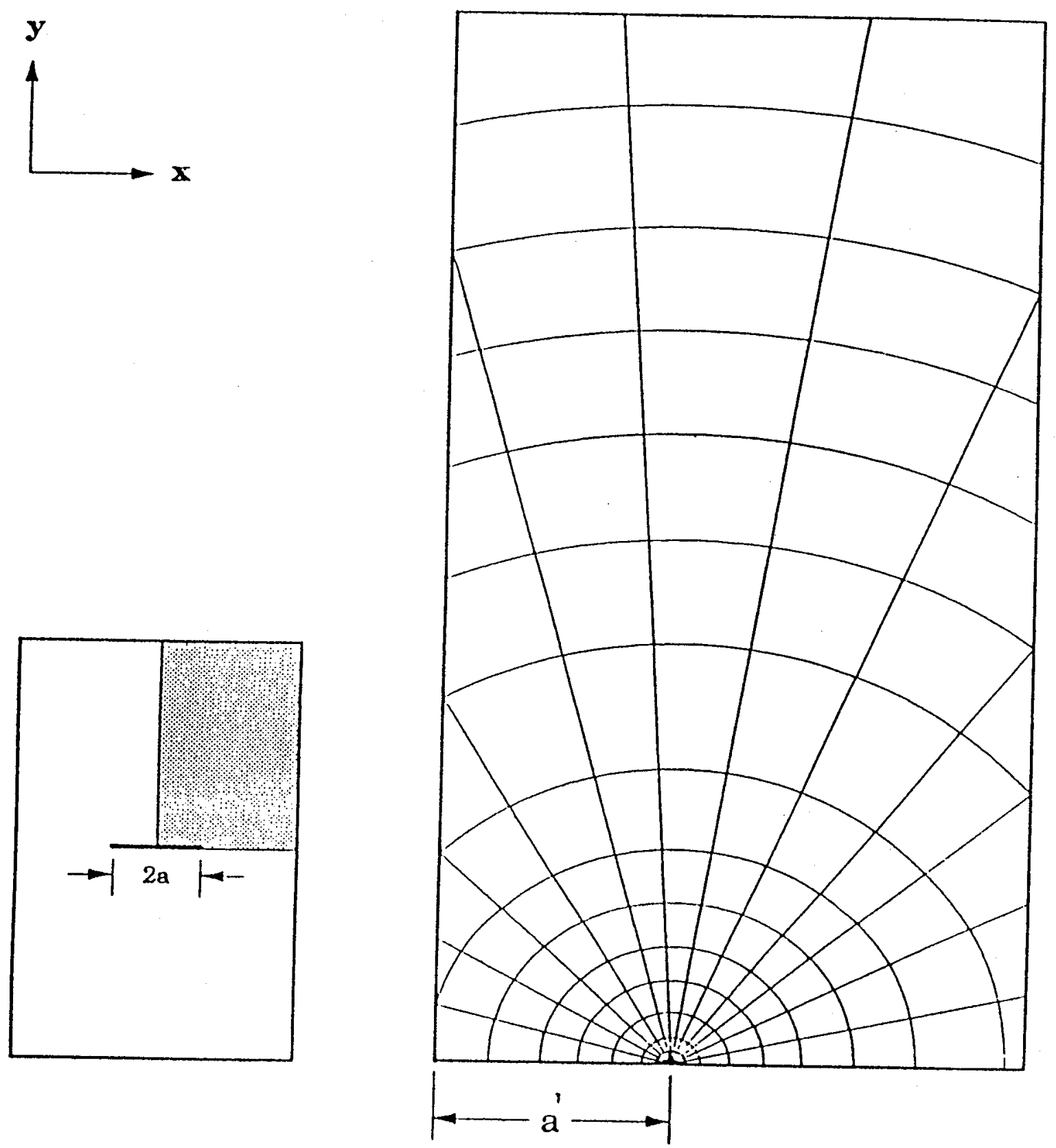

Fig. 2. The finite element mesh. For the single-edge-cracked case, $a$ is the crack length, whereas for the center-cracked case, $a$ is the half-length. 

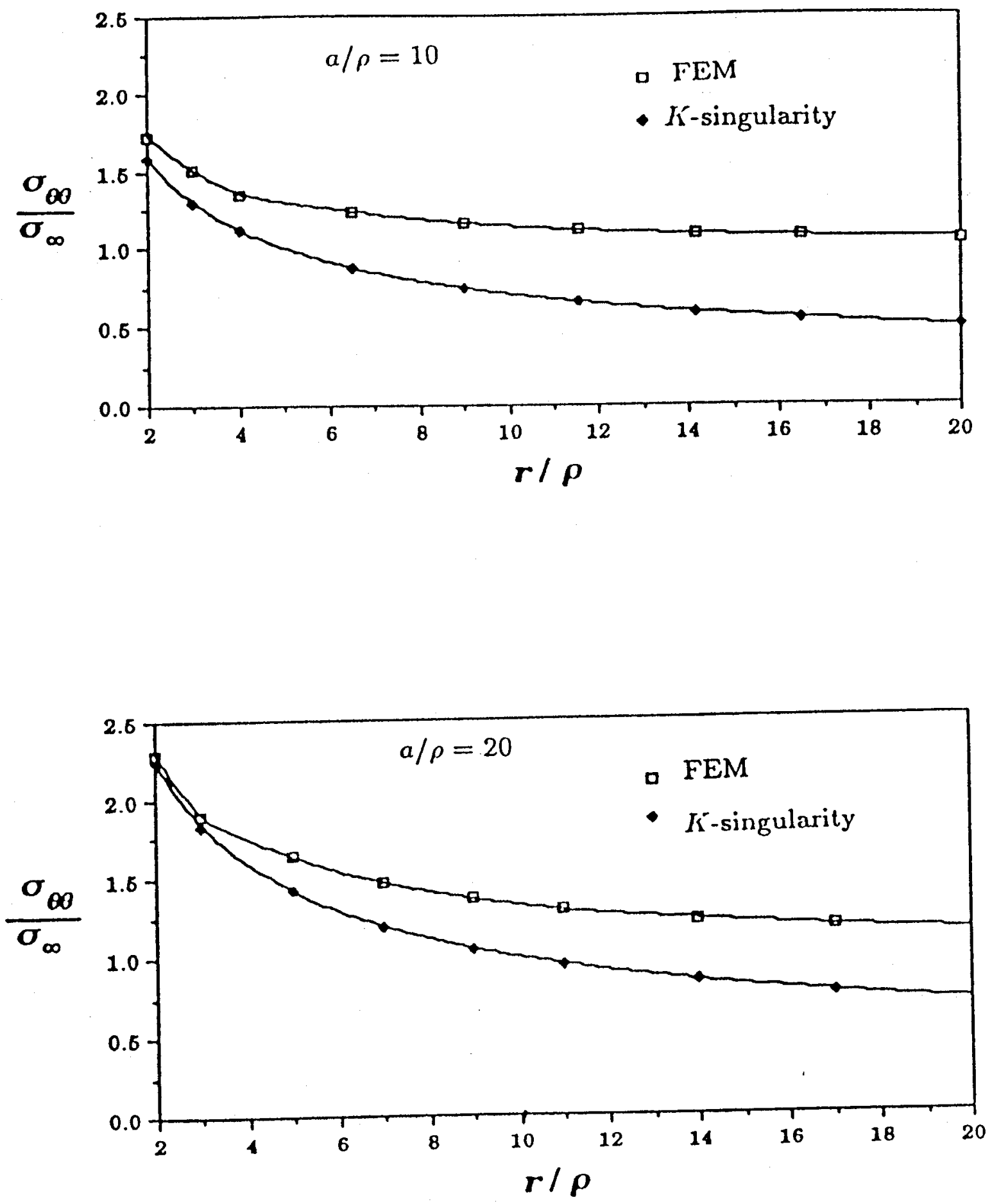

Fig. 3a. The stress field $\sigma_{\theta \theta}$ directly ahead of the crack tip for $a / \rho=10,20$ in the center-cracked specimen. 

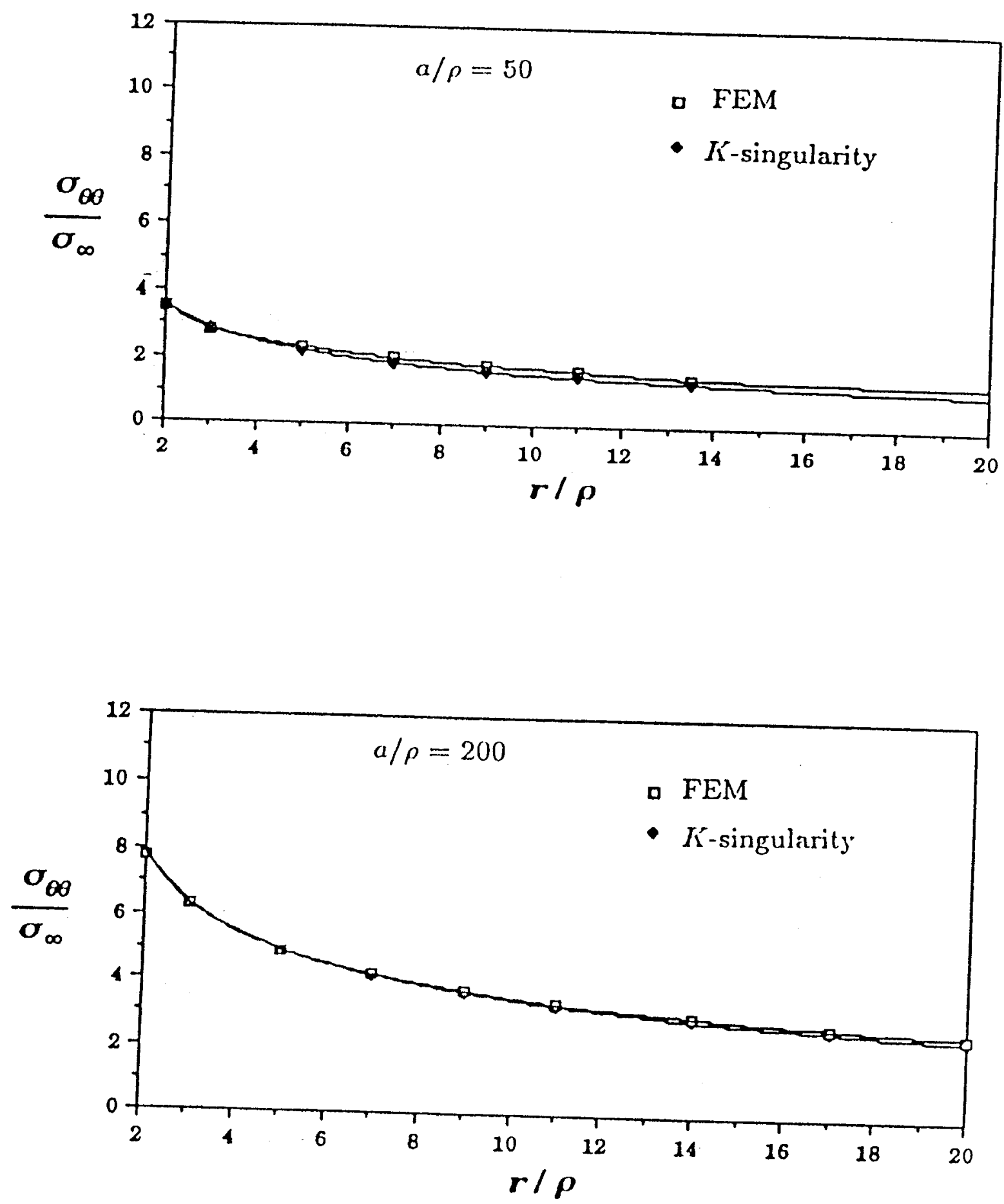

Fig. 3b. The stress field $\sigma_{00}$ directly ahead of the crack tip for $a / \rho=50,200$ in the centercracked specimen. 

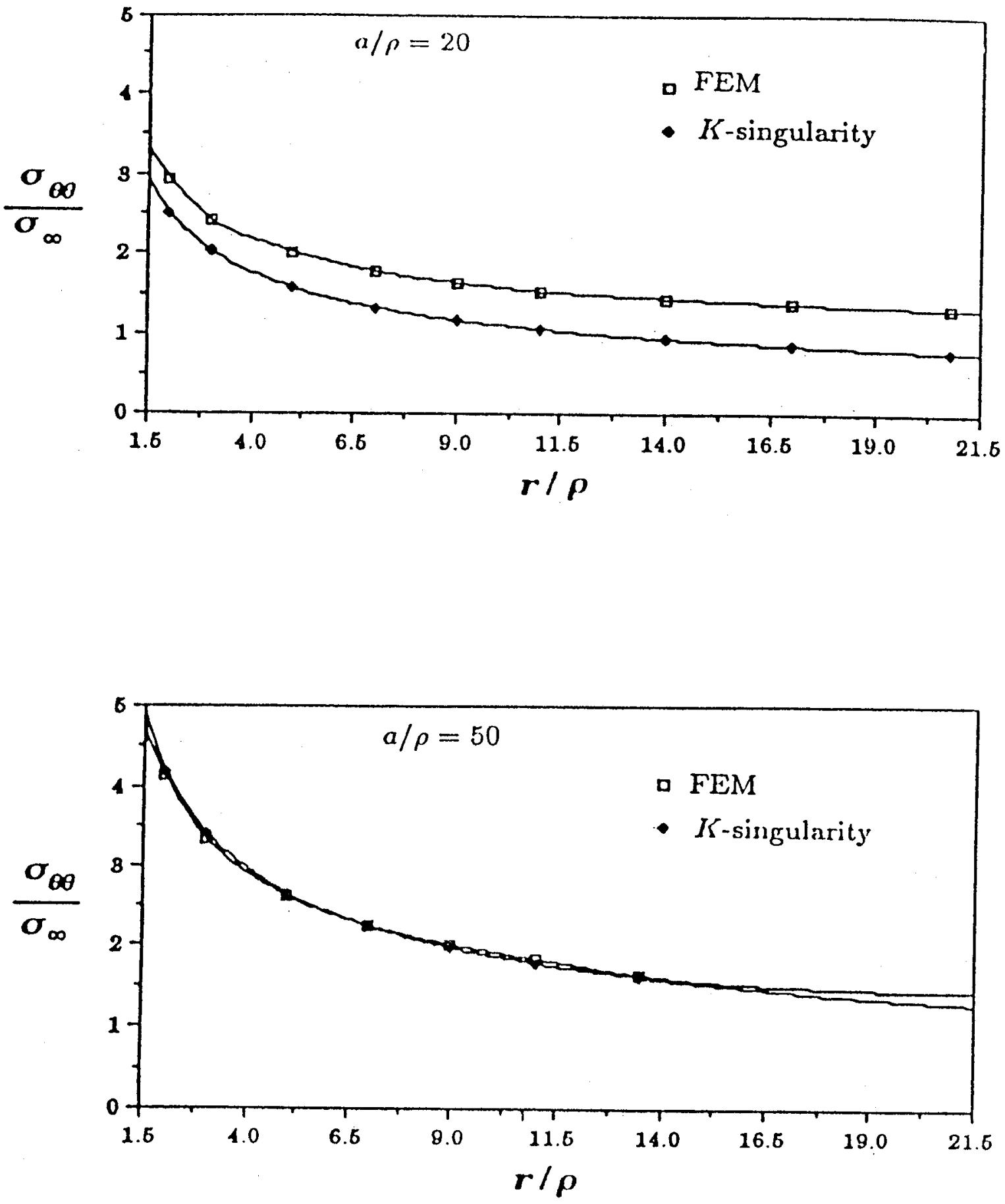

Fig. 4. The stress field $\sigma_{\theta \theta}$ directly ahead of the crack tip for $a / \rho=20,50$ in the single-edgecracked specimen. 


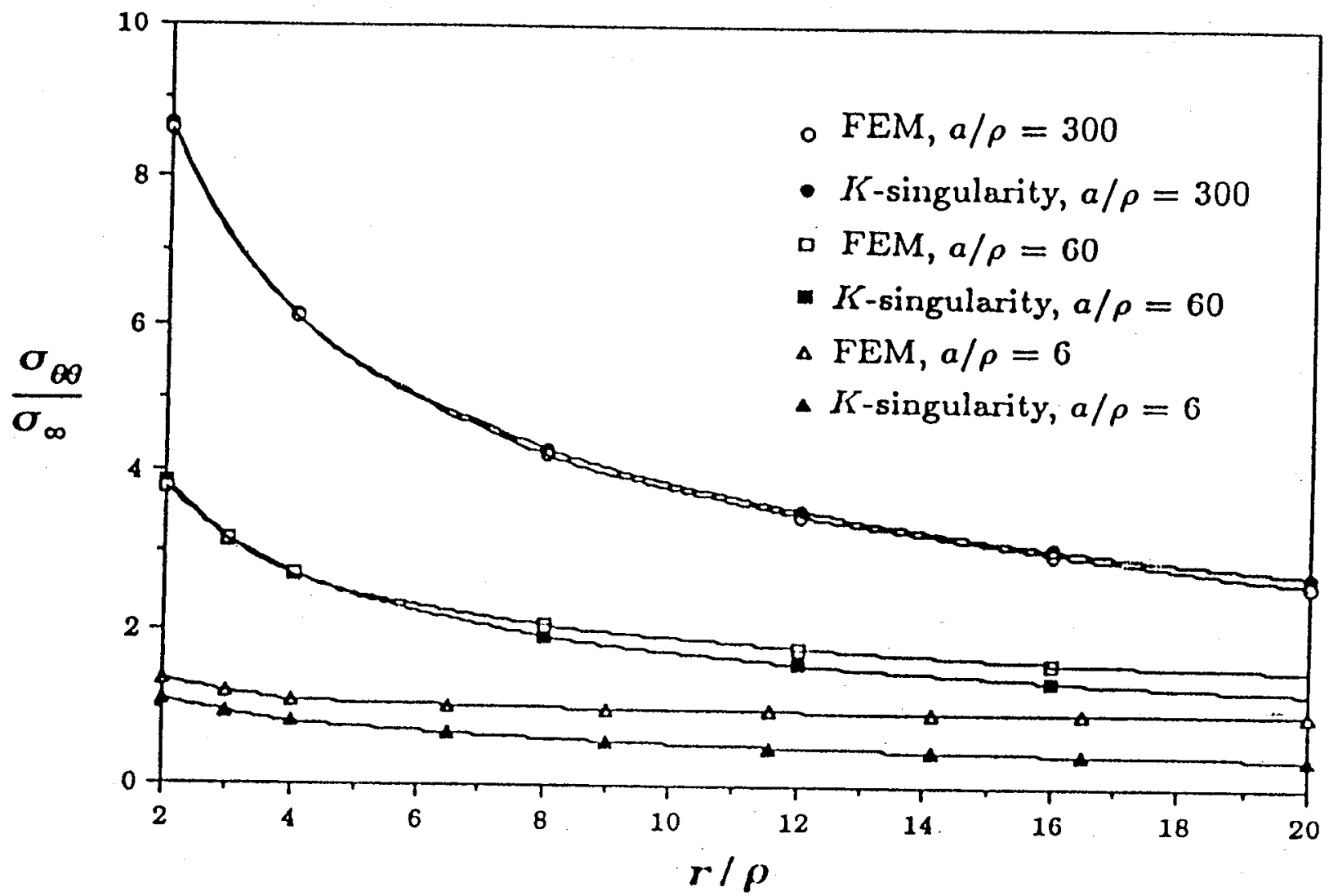

Fig. 5. The stress field $\sigma_{0 \theta}$ directly ahead of the crack tip for three center-cracked specimens which have the same $a / w$ ratio of 0.02 but different absolute sizes (i.e. a sliort crack, $a / \rho=6$, and two long cracks, $a / \rho=60$ and 300$)$. 


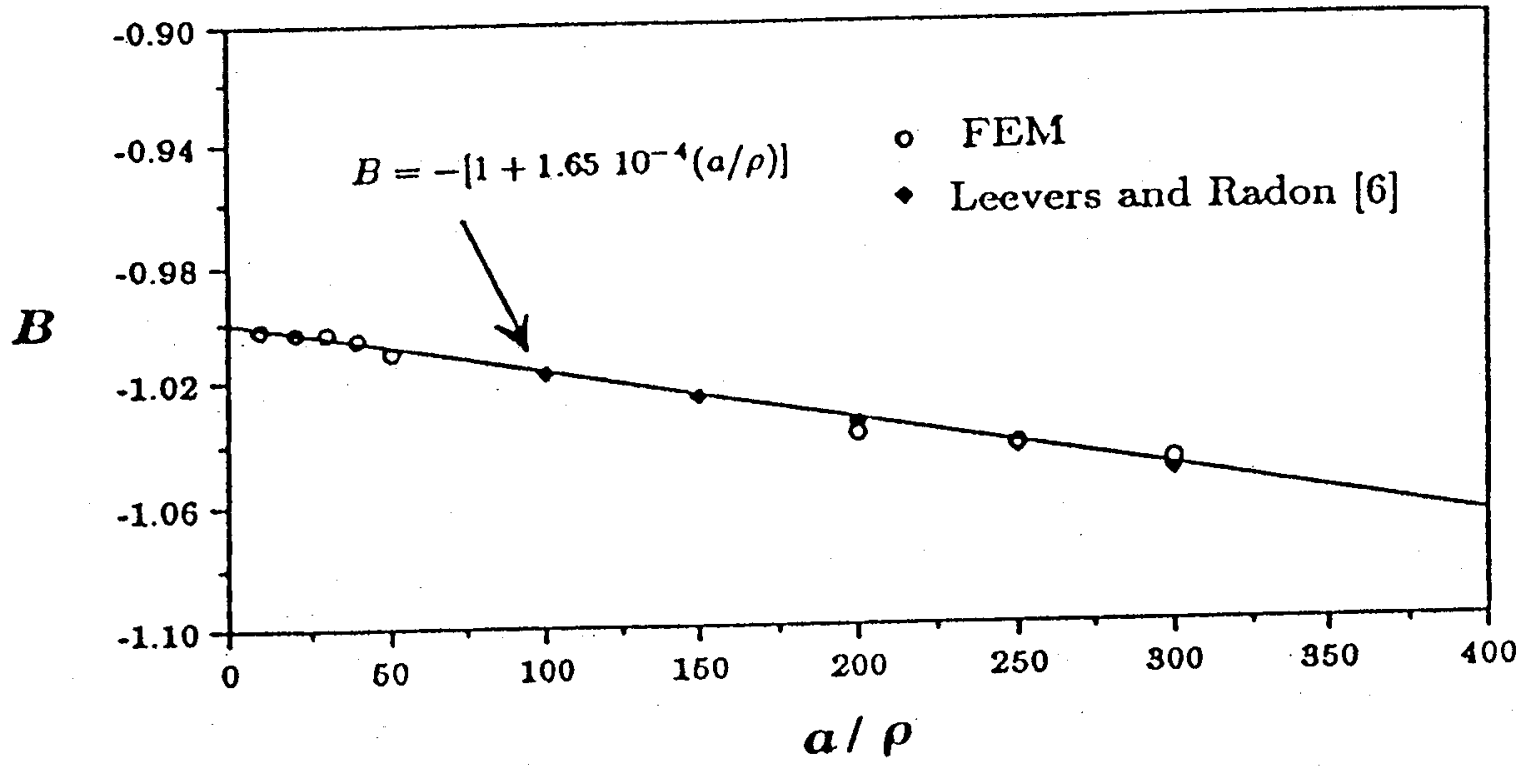

(a)

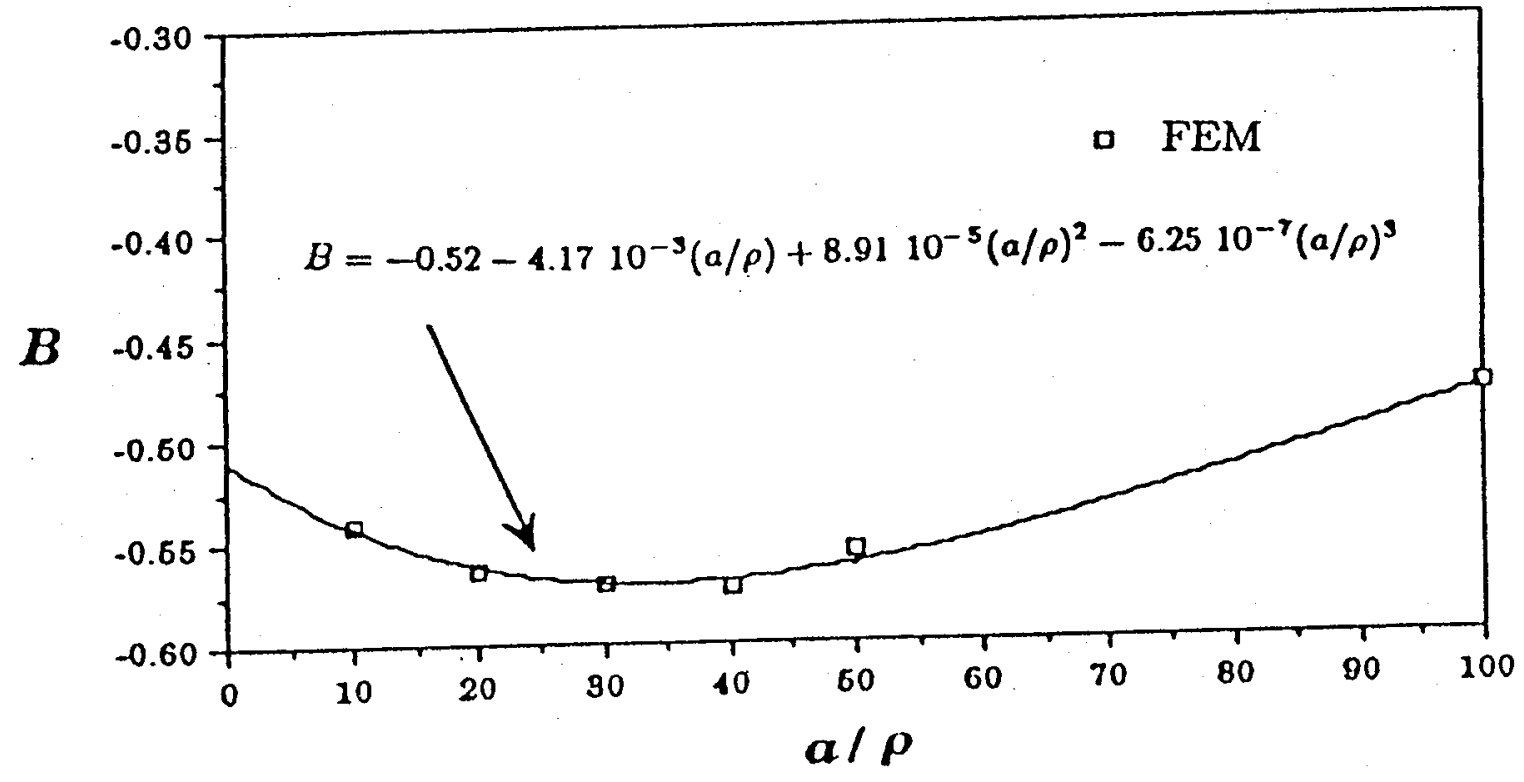

(b)

Fig. 6. The biaxiality ratio, $B$ as a function of crack length $a$ for (a) the center-cracked and (b) the single-edge-cracked specinens. 


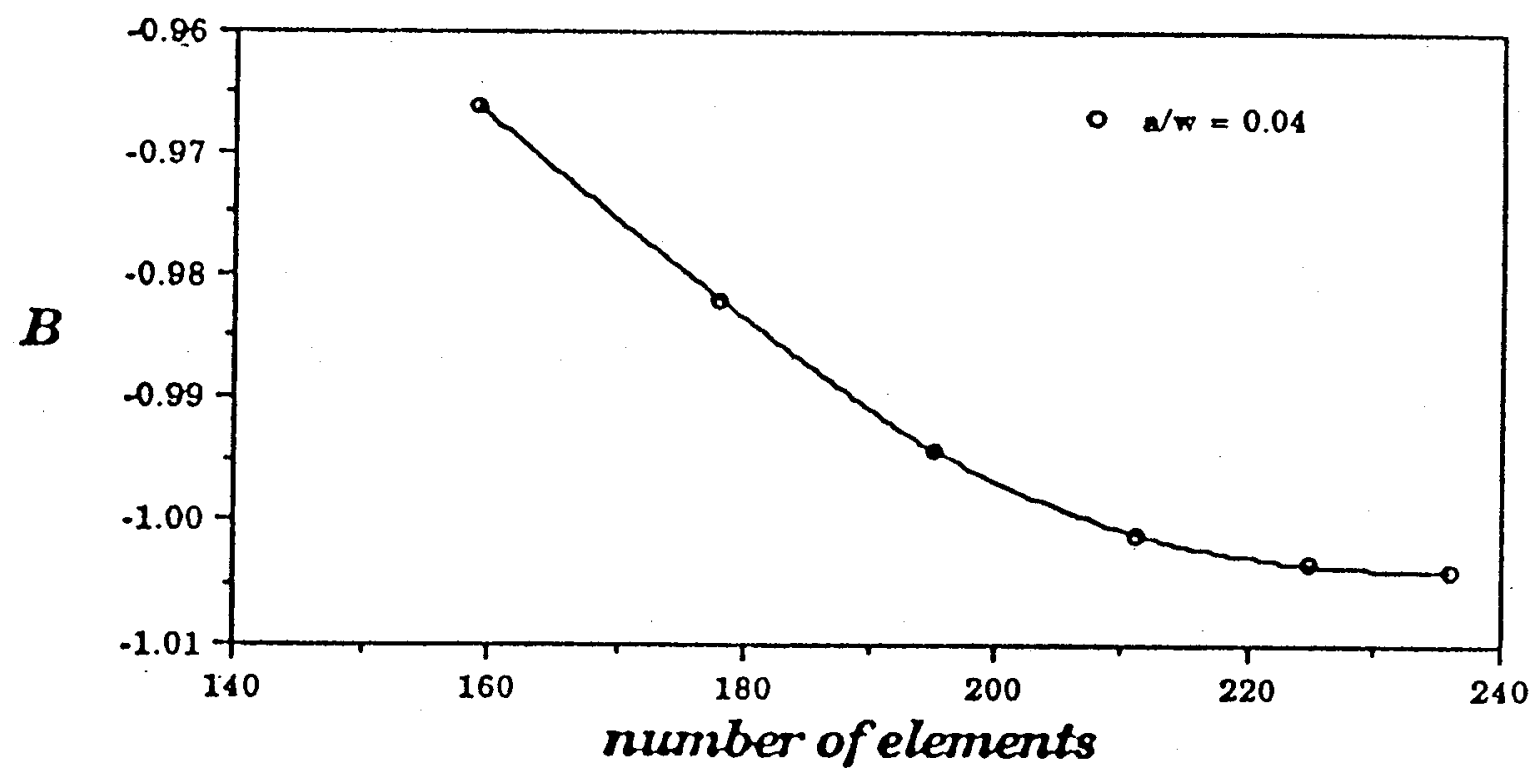

Fig. 7. The biaxiality ratio, $B$ as a function of the number of elements, illustrating the sensitivity of the numerical scheme. 

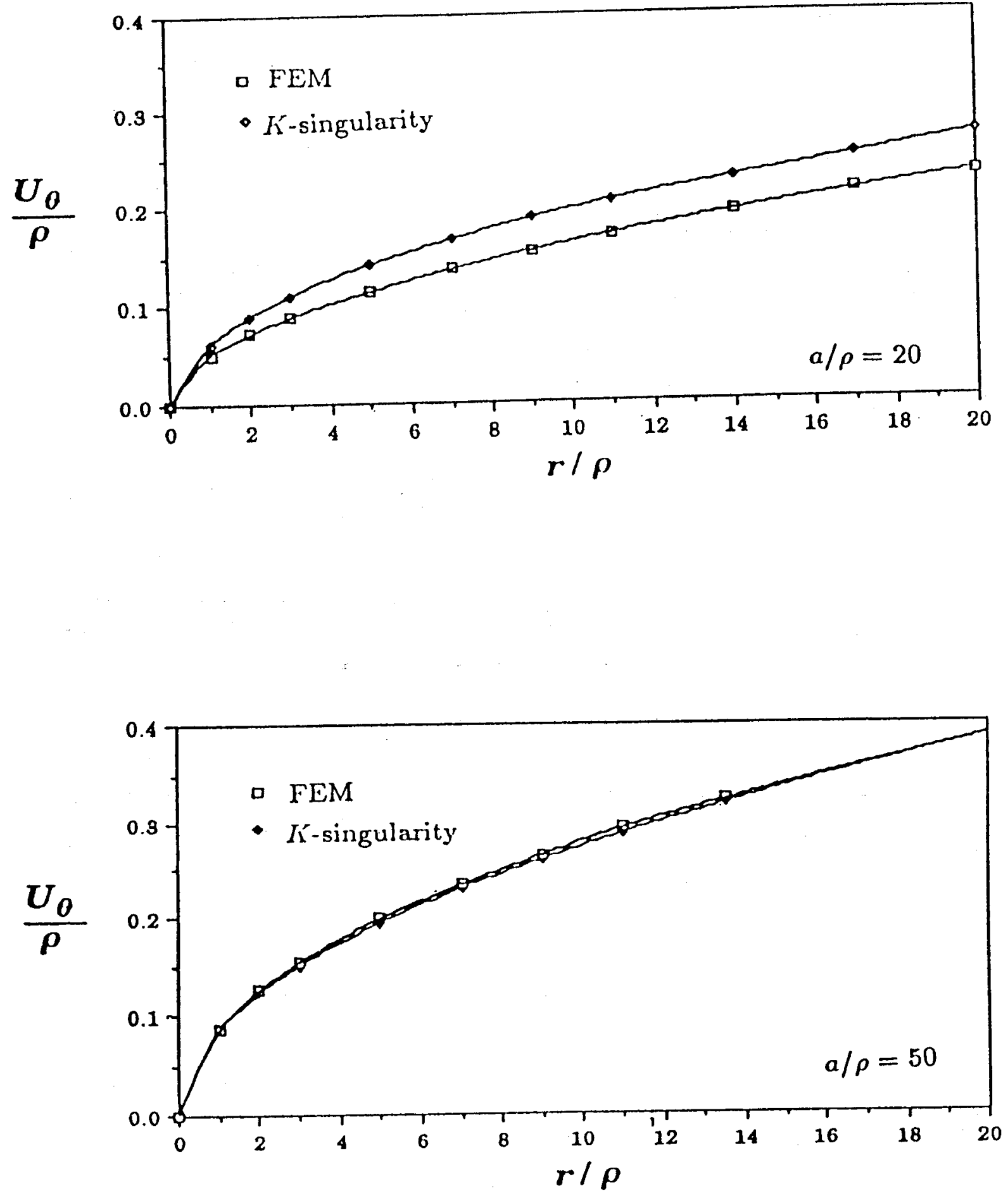

Fig. 8. The displacement field $U_{0}$ behind the crack tip for $a / \rho=20,50$ in the single-cdgecracked specimen. 

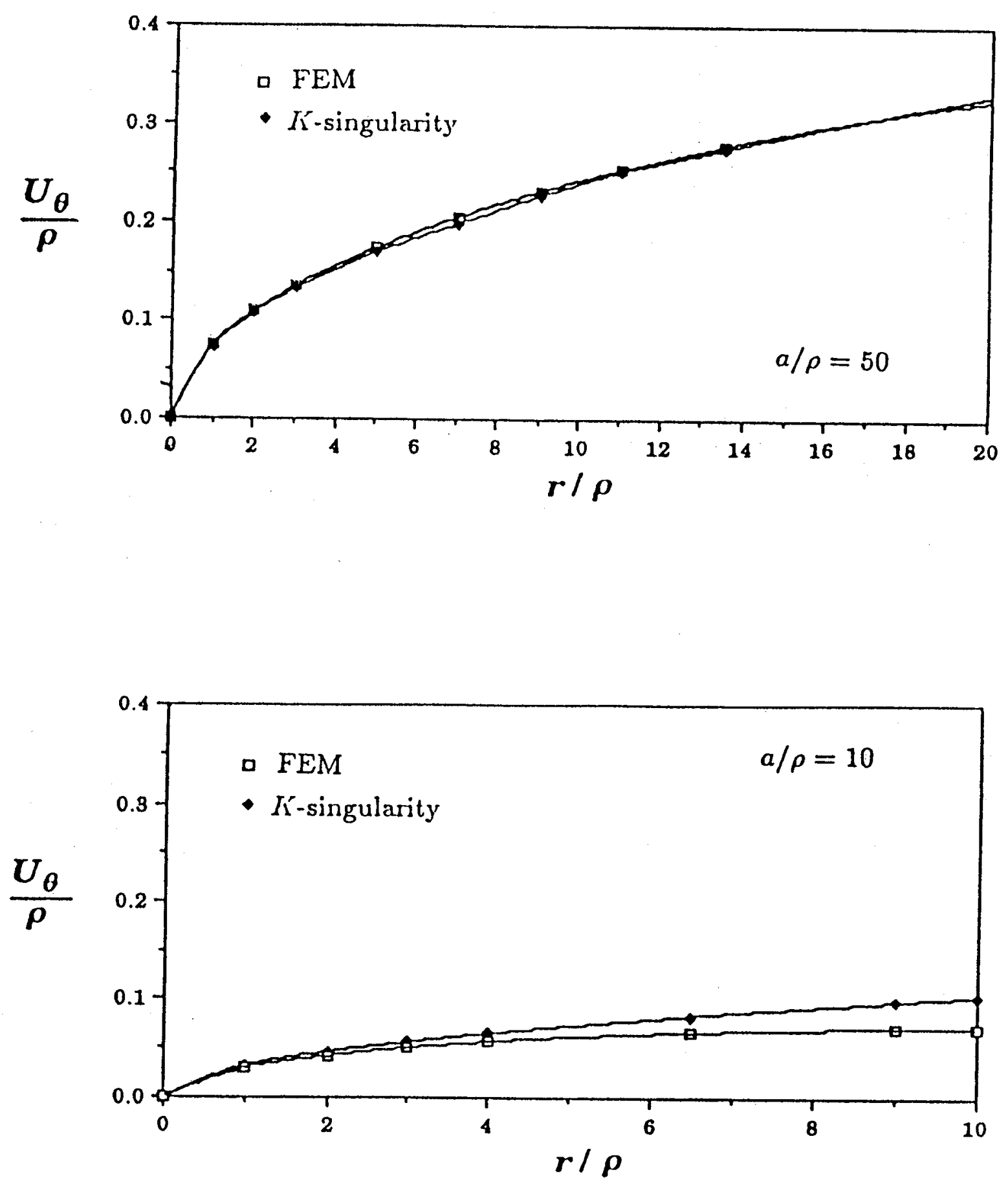

Fig. 9. The displacement field $U_{0}$ behind the crack tip for $a / \rho=10,50$ in the center-cracked specimen. 


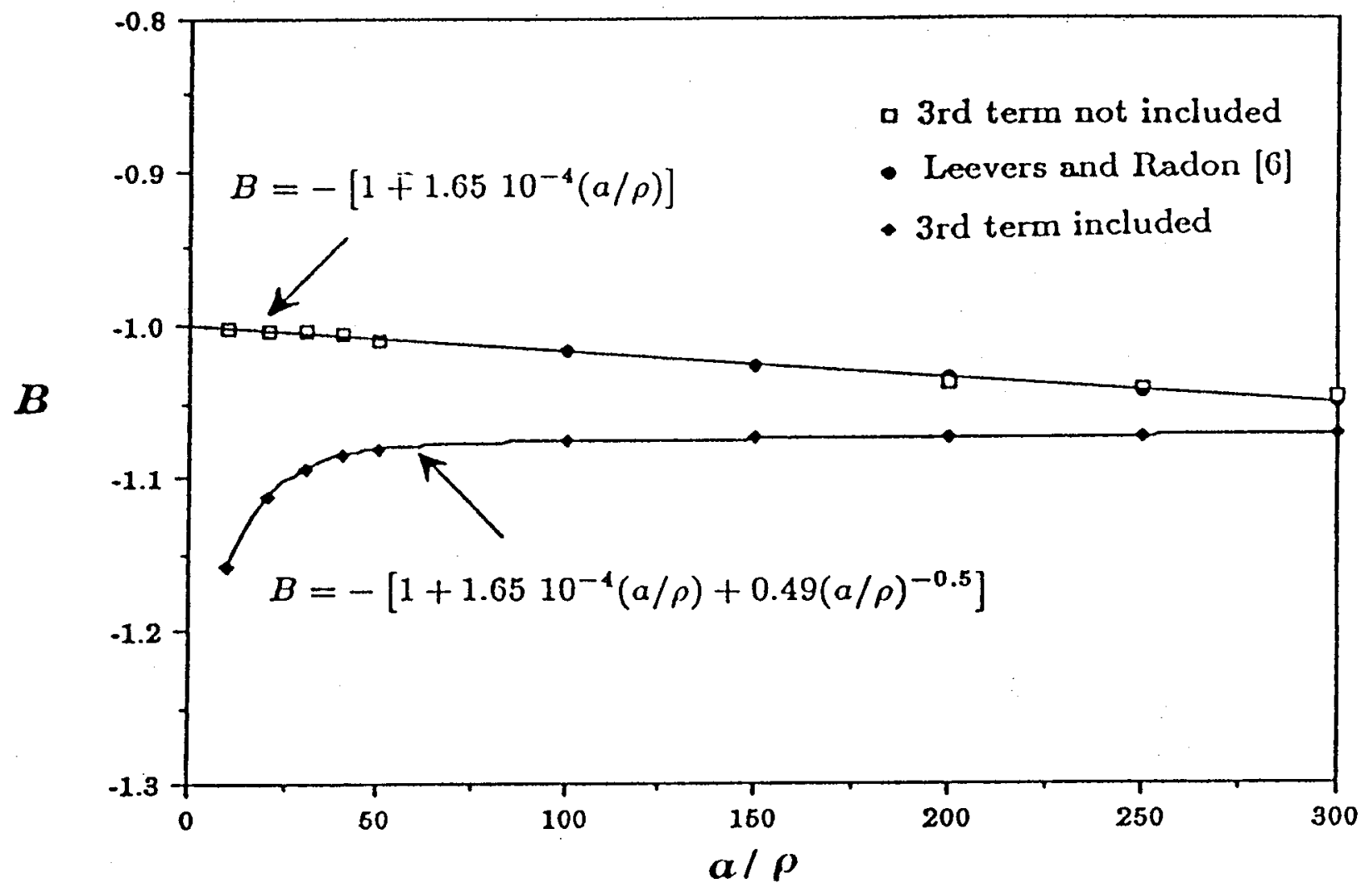

Fig. 10. The effect of the third term on the biaxiality ratio for the center-cracked geometry. 


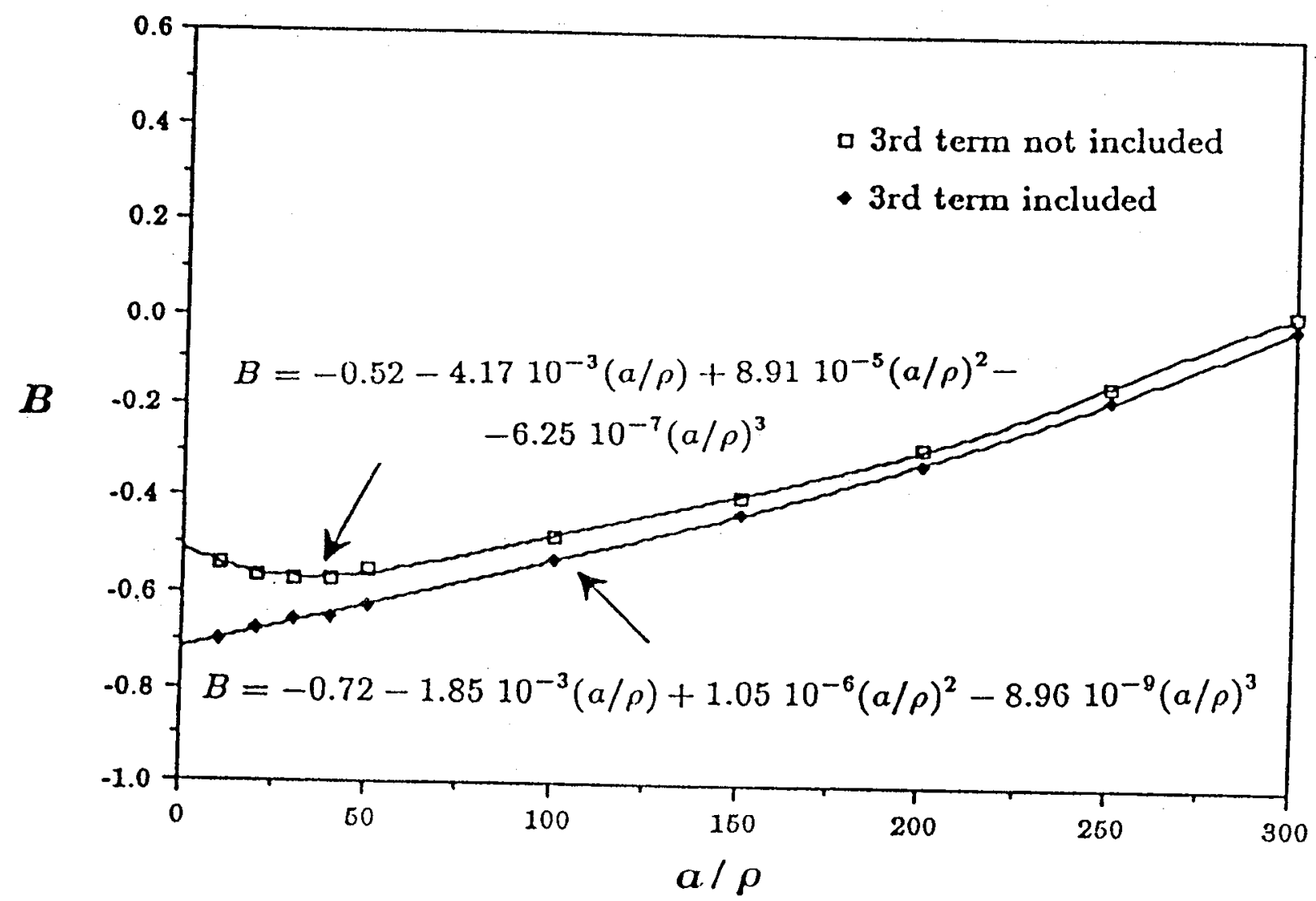

Fig. 11. The effect of the third term on the biaxiality ratio for the single-edge-cracked geometry. 


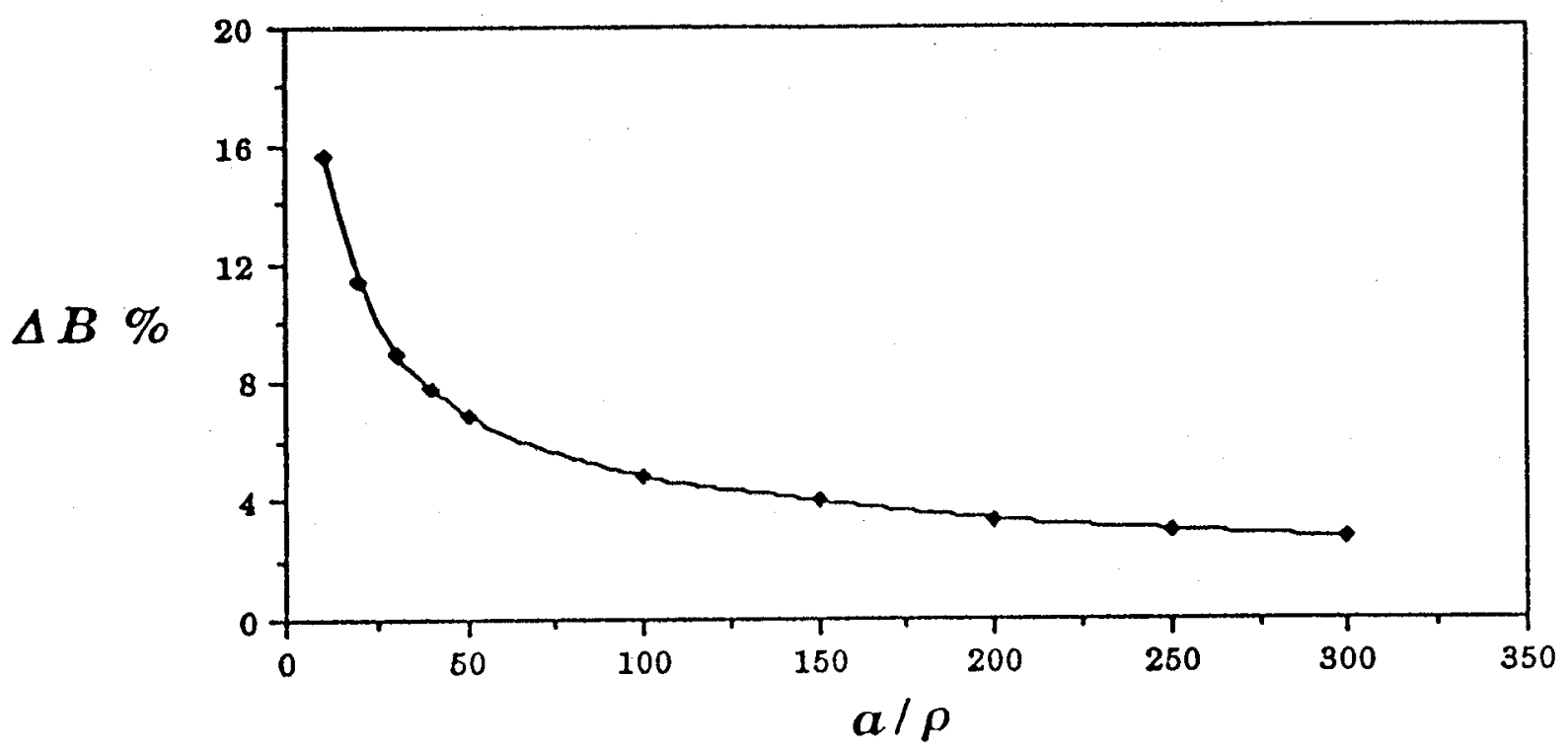

Fig. 12. Percentage increase in $B, \Delta B=\left(B_{\text {incl }}-B_{\text {not incl }}\right) / B_{\text {not incl }}$ in the center-cracked case. 